\title{
Improving the safety of prescriptions of domperidone in primary care: implementing MHRA advice.
}

\author{
William Hall \\ Holland Park Surgery, London
}

\begin{abstract}
Domperidone is a dopamine D2 receptor antagonist acting on the chemoreceptor trigger zone in the medulla and also in the gut, causing antiemetic and gastrokinetic effects respectively. In the past, domperidone was considered largely safe, with many indications and few contraindications listed in the product literature. In 2014, Domperidone became indicated only for the prevention of nausea and vomiting and the duration of treatment was limited to seven days. Furthermore, the maximum daily dose was limited to thirty milligrams. A quality improvement project was undertaken at Holland Park Surgery to improve compliance with MHRA guidelines. Prescriptions of domperidone in the previous nine months were assessed for compliance with the MHRA advice. Domperidone was prescribed for 23 patients; of these 4 were single acute prescriptions, 3 were repeats which had been stopped and 16 were on active repeat at the time of the search. All patients who had active repeat prescriptions had exceeded the recommended duration of treatment. MHRA contraindications were found in $6(37 \%)$ of active repeat prescriptions. The strategy for improvement involved three PDSA cycles and involved engaging with patients for medication reviews and staff to improve prescribing practices. After the third PDSA cycle we demonstrated that all repeat prescriptions had been stopped and that new prescriptions were compliant with MHRA advice.
\end{abstract}

\section{Problem}

Domperidone is a dopamine D2 receptor antagonist working at the chemoreceptor trigger zone and in the gut, causing antiemetic and gastrokinetic effects respectively. Domperidone has been under surveillance in the EU after concerns regarding serious adverse cardiac events.[1] The Medicines and Healthcare products Regulatory Agency (MHRA) published new advice on the use of domperidone in 2014 which provided new advice on its safe use.[2] The local Clinical Commissioning Group disseminated this information to GP practices.

We decided to undertake a quality improvement project at Holland Park Surgery in West London. Holland Park Surgery is located in the Borough of Kensington and Chelsea and serves a diverse community of patients. Kensington and Chelsea has proportionally lower rates of long term limiting illness compared to national figures and is ranked top nationally for residents who assess their health as "very good". The local population has comparable unemployment rates to the national average. Over half of residents have a qualification equivalent to a bachelor's degree, the fourth highest in England.[3] The project was designed and coordinated by the author with advice and guidance from GPs who knew the patients involved.

The aim of this project was to improve the compliance of domperidone prescriptions with MHRA advice. This would be through the reduction of unnecessary and non-compliant prescriptions to $0 \%$ over a three month period.

\section{Background}

In the 1980's concerns were raised about possible adverse cardiac events associated with the use of high doses of intravenous domperidone, which was being used as an antiemetic during chemotherapy. Of particular concern was the risk of QTc interval prolongation, ventricular arrhythmia, and sudden cardiac death. The intravenous preparation was subsequently withdrawn from use; however, until recently the oral preparation had come under less scrutiny. Prior to recent European Medicines Agency (EMA) and MHRA advice, domperidone had wide range of indications beyond nausea and vomiting, which included delayed gastric emptying, heart burn, and regurgitation.[1]

In 2014 the EMA's Pharmacovigilance Risk Assessment Committee compiled an assessment report on the safety of Domperidone.[1] The assessment concluded that domperidone is associated with a small increased risk of serious cardiac adverse events, including QTc interval prolongation and sudden cardiac death. A higher risk was associated with patients over the age of 60 , those taking daily domperidone doses of more than $30 \mathrm{mg}$, and those taking medicines that also cause QTc prolongation or inhibit CYP3A4. The MHRA now recommends that domperidone is only used for the short term treatment of nausea and vomiting. The maximum adult daily dose should not exceed thirty milligrams and the duration of treatment should not exceed seven days.[2] Domperidone is now contraindicated in people with conditions where cardiac conduction is, or could be, impaired; in people with underlying cardiac diseases such as congestive heart failure; in people receiving other medications known to prolong QT interval or potent CYP3A4 inhibitors; in those with severe hepatic impairment.

\section{Baseline measurement}

"SystemOne" is an electronic patient record and prescription system 
designed for primary care and was in use at Holland Park Surgery. All prescriptions for domperidone from the previous nine months were collated. During this period domperidone was prescribed for twenty-three patients $(n=23)$; four were single acute prescriptions, three were repeat prescriptions that had been stopped, and sixteen were active repeat prescriptions at the time of the search. Nausea and Vomiting was the recorded indication for $13(57 \%)$ of total prescriptions, with other indications including: bloating, gastritis, regurgitation, poor motility, reflux, and cramping. Half of acute prescriptions met the MHRA guidelines for duration but all repeat prescriptions exceeded the recommended duration. There were no contraindications found for acute single prescriptions; however, active repeat prescriptions demonstrated that six (37\%) of patients had MHRA listed contraindications. Four were because the patient was taking other medications known to prolong QTc interval, specifically citalopram, fluoxetine, and amitriptyline. One had a known cardiac conduction abnormality and one had underlying cardiac disease. Two patients were taking doses above safe recommendations. In summary, all sixteen repeat prescriptions were not compliant with MHRA advice and six had contraindications.

See supplementary file: ds7232.pdf - "Graphs of baseline data."

\section{Design}

It was noted that all repeat prescriptions for domperidone had been created prior to new guidance, but had not be reviewed since. We observed that repeat prescriptions were being re-authorised at the patient's request by administration staff or doctors. These were seen as missed opportunities for medication review and intervention. Prescribing staff reported they felt it was a challenge to stop a long term medication in a patient they had just met, especially when the patient wished to continue the medication.

There were two priorities in our approach to improving compliance with MHRA guidelines. Firstly to perform medication reviews for all patients taking domperidone. Initially this was done electronically by the author and the patient's general practitioner. An individual action plan for the next step of the medication review was then developed for each patient. Secondly, to ensure that future prescriptions of domperidone were compliant, we planned to engage doctors and administration staff through practice meetings, teaching, and via email communications. The MHRA guidelines were presented to the team at the practice multidisciplinary meeting to update all staff. The PDSA cycle was used as a tool to guide the improvement process.

\section{Strategy}

PDSA cycle one:

Medication reviews for all those patients found to be on repeat prescriptions of domperidone were commenced. The author and the patient's own general practitioner performed 'virtual' medication reviews using the electronic patient record system. Individual action plans were devised, outlining how to best engage with each patient.
For six patients a letter was composed explaining the new guidelines and included a copy of the MHRA letter to patients. The patients were also invited to book a telephone or face to face consultation to discuss the matter further if they wished. Three of these patients decided to have telephone follow up. Ten patients were contacted by telephone in the first instance and had a medication review performed. Two patients had received telephone medication reviews but were reluctant to stop taking domperidone. The medication had been started in secondary care nearly ten years ago and the patients were concerned they may have a worsening of their chronic conditions if it was stopped abruptly. Both patients were taking domperidone for gastro-oesophageal reflux. One patient was taking two other medications known to prolong QTc interval and we were particularly concerned at this. This was discussed with the pharmacist who recommended domperidone should not be taken in this circumstance. These cases were discussed at a practice meeting between the senior general practitioners and the practice pharmacist.

PDSA cycle two: Both of these two patients had further telephone consultations with their general practitioner and the author, after which they agreed to wean their use of domperidone. Both repeat prescriptions were subsequently stopped. After one month, the clinical records were analysed once again. It was identified that an acute prescription had been issued for 120 tablets of domperidone to a patient who had agreed to have their repeat prescription stopped. We recognised that a patient had visited a different GP and requested further domperidone, and despite having contraindications was issued a prescription.

PDSA cycle three:

In reaction to this prescription we decided to carry out a teaching session for all general practitioners, trainees, and administration staff to highlight the changes to the guidelines for the safe prescribing of domperidone. The guidelines were also emailed to all those who work in the practice. After these interventions a two month period of further data collection took place. After this time, no repeat prescriptions existed and the only acute prescription for domperidone was identified and was compliant with MHRA guidelines.

See supplementary file: ds7526.pdf - "Number of non-compliant prescriptions for domperidone over time "

\section{Post-measurement}

After three PDSA cycles, all prescriptions of domperidone were compliant with MHRA guidelines. No repeat prescriptions existed and a single acute prescription was compliant. Staff reported that they were now aware of the guidelines and that they were implementing this in their prescribing practice. This was supported by a further period of data collection.

\section{Lessons and limitations}

This project highlighted the importance of ensuring that medication 
safety alerts are integrated into practice. Despite the MHRA advice on domperidone being published in April 2014, a review had not yet been carried out and many staff were not aware of the new guidelines. It was a learning point for the practice that regular audits need to be carried out to ensure new guidelines are acted upon. Systems are now being developed to ensure that future medication alerts are disseminated within the practice. Prescriptions of domperidone were often started before new guidelines were published and a minority of patients were reluctant to now stop the medication. This led to a dilemma of continuing to prescribe medication when the patient is aware of the risks, and our responsibility as safe prescribers. In these situations the practice pharmacist was helpful and senior general practitioners who had good rapport with the patients were able to negotiate satisfactory and safe outcomes. This demonstrated the importance of local knowledge and experience in addressing problems.

This audit was limited to primary care and prescribing practices of domperidone in secondary care is likely to differ and possible pose differing challenges. However, this audit has demonstrated that the use of domperidone may not always reflect the latest guidelines and must be reviewed across all clinical areas. Whilst changes in prescribing knowledge and behaviour were not specifically studied, the outcome has demonstrated changes in prescribing practice. The sustainability of this intervention can be challenged on this basis. Further work should address the complexity of prescribing practice by improving systems which will encourage better prescribing practices overall. Despite this, this project demonstrates a useful framework which can be utilised by others to address specific focused prescribing concerns.

\section{Conclusion}

This project demonstrated that prescribing practices of domperidone were sub-optimal, with many prescriptions being noncompliant with new MHRA safety advice. Due to the risk of cardiac side effects of this medication if used incorrectly, it was deemed a patient safety issue which needed to be addressed. This audit identified prescriptions not compliant with the MHRA guidance and took steps to ensure prescriptions of domperidone were reviewed and stopped if appropriate. Furthermore, education led to better awareness amongst prescribing staff of the updated guidelines and led to improved prescribing behaviours. Changing professional behavior is a complex process and conclusions on how best to do this are difficult to draw upon (4); however, this project has demonstrated an approach to address a specific prescribing problem. With a relatively small sample size, an individual approach to each patient was possible and the multidisciplinary team was involved. This represents a reproducible project which can be implemented in other primary care settings.

\section{References}

1. European Medicines Agency. Pharmacovigilance Risk Assessment Committee (PRAC), Assessment report. Domperidone-containing medicinal products, 2014. Available from: http://www.ema.europa.eu/docs/en GB/document library/R eferrals_document/Domperidone_31/Recommendation_pro vided_by_Pharmacovigilance_Risk_Assessment_Committe e/WC500168926.pdf

2. Medicines and Healthcare products Regulatory Agency. Domperidone: risks of cardiac side effects. Drug Safety Update. 2014; 7(10). Available from: https://www.gov.uk/drug-safety-update/domperidone-risks-ofcardiac-side-effects

3. Census (2011): Kensington and Chelsea. A summary of all Key Statistics and a selection of Quick Statistics from the second release of Census statistics (11 December, 2012). Available from: https://goo.gl/cwbRkm

4. Robertson R. and Jochelson K. (2006): Interventions that change clinician behaviour:

mapping the literature. King's Fund, London. Available at: https://goo.gl/KVep8k

\section{Declaration of interests}

None declared.

\section{Acknowledgements}

Dr. Bavani Dharmawardene

\section{Ethical approval}

This was deemed a quality improvement project by West London Clinical Commissioning Group and the partners of Holland Park Surgery. Ethical approval was not required. 